\title{
Security of an Identity-Based Cryptosystem and the Related Reductions ${ }^{\star}$
}

\author{
Tatsuaki Okamoto Shigenori Uchiyama \\ NTT Laboratories \\ 1-1 Hikarinooka, Yokosuka-shi, 239-0847 Japan \\ Email: \{okamoto, uchiyama\}@sucaba.isl.ntt.co.jp
}

\begin{abstract}
Recently an efficient solution to the discrete logarithm problem on elliptic curves over $F_{p}$ with $p$ points ( $p$ : prime), so-called anomalous curves, was independently discovered by Semaev [14], Smart [17], and Satoh and Araki [12]. Since the solution is very efficient, i.e., $O\left(|p|^{3}\right)$, the Semaev-Smart-Satoh-Araki (SSSA) algorithm implies the possibility of realizing a trapdoor for the discrete logarithm problem, and we have tried to utilize the SSSA algorithm for constructing a cryptographic scheme. One of our trials was to realize an identity-based cryptosystem (key-distribution) which has been proven to be as secure as a primitive problem, called the Diffie-Hellman problem on an elliptic curve over $\mathrm{Z} / n \mathrm{Z}\left(\boldsymbol{n}=p q, p\right.$ and $q$ are primes) where $E_{p}$ and $E_{q}$ are anomalous curves (anomalous $E_{n}$-Diffie-Hellman problem). Unfortunately we have found that the anomalous $E_{n}$-Diffie-Hellman problem is not secure (namely, our scheme is not secure). First, this paper introduces our trial of realizing an identity-based cryptosystem based on the SSSA algorithm, and then shows why the anomalous $E_{n}$-Diffie-Hellman problem is not secure. In addition, we generalize the observation of our breaking algorithm and present reductions of factoring $n$ to computing the order ${ }^{2}$ of an elliptic curve over $\mathbf{Z} / n \mathbf{Z}$. (These reductions roughly imply the equivalence of intractability between factoring and computing elliptic curve's order.) The algorithm of breaking our identity-based cryptosystem is considered to be a special case of these reductions, and the essential reason why our system was broken can be clarified through these reductions: $E_{n}$ in our system is a very specific curve such that the order of $E_{n}$ (i.e., $n$ ) is trivially known.
\end{abstract}

\section{Introduction}

\subsection{Background}

One of the most interesting open problems in cryptography is the realization of a trapdoor on the discrete logarithm, in which to solve the discrete logarithm is hard only if published parameters are used, while it is easy by using a secret key (trapdoor key).

One of the possible candidates to solve the problem is: a pair of primes, $(p, q)$, is a secret key, and $n=p q$ is the corresponding public-key. (1) the discrete

\footnotetext{
* This is an updated version. The original version accepted to the conference had a security weakness.

${ }^{2}$ We define the order of an elliptic curve, $E_{n}$, over $\mathbf{Z} / n \mathrm{Z}$ by $\operatorname{lcm}\left(\# E_{p}\right.$, \# $\left.E_{p}\right)$, where $n=p q$ ( $p, q$ : primes), $E_{p}$ and $E_{q}$ are elliptic curves over $\mathbf{F}_{p}$ and $\mathbf{F}_{q}$, and $E_{n}$ is the Chinese remainder composite of $E_{p}$ and $E_{q}$
} 
logarithm $\bmod n$ is intractable against an adversary who knows no factors of $n$ and (2) a person who knows a trapdoor, $(p, q)$, can compute the discrete logarithm $\bmod n$ efficiently.

However, this trapdoor trick does not seem to work well. This is because: in order to make the trapdoor for computing the discrete logarithm $\bmod n$ efficiently (i.e., to make the discrete $\operatorname{logarithm} \bmod p$ be easy), $p-1$ should consist of all (fairly) small primes. Such $n$ is (fairly) easily factored by the $p-1$ method. Of course, we can make some delicate computational complexity gap between computing the discrete $\log$ arithm $\bmod p$ and factoring $n[7,8]$, but it seems hard to make a computationally feasible gap such that one is a polynomial time and the other an (assumed) super-polynomial time.

\subsection{New hope: anomalous elliptic curves}

The discrete logarithm problem can be defined on various finite groups as well as the multiplicative group over a finite field $\mathbf{F}_{q}$. A typical example except the multiplicative group is the discrete logarithm problem on elliptic curves over $\mathbf{F}_{q}$, and many cryptographic schemes are constructed on the elliptic curve discrete logarithm problems.

Recently an efficient solution of the discrete logarithm problem on elliptic curves over $\mathbf{F}_{p}$ with $p$ points ( $p$ : prime), so-called anomalous curves, was independently discovered by Semaev [14], Smart [17], and Satoh and Araki [12]. The solution is very efficient, i.e., $O\left(|p|^{3}\right)$, and is almost as efficient as typical practical public-key encryption and decryption algorithms such as those of the RSA scheme. The Semaev-Smart-Satoh-Araki (SSSA) algorithm, therefore, implies the possibility of realizing a trapdoor for the discrete logarithm problem on elliptic curves over $\mathbf{Z} / n \mathbf{Z}$, (say $E_{n}$ ) where $E_{n}$ is the Chinese remainder composition of anomalous elliptic curves, $E_{p}$ and $E_{q}$. Here, the discrete logarithm over $E_{n}$ seems hard without knowing $(p, q)$, while if the trapdoor information $(p, q)$ is known, it can be easily calculated by using the SSSA algorithm for $E_{p}$ and $E_{q}$.

\subsection{Identity-based cryptosystems}

We have tried to utilize this trapdoor trick based on the Semaev-Smart-SatohAraki (SSSA) algorithm for realizing a cryptographic scheme. One of our trials was to realize an identity-based cryptosystem (key-distribution).

In 1984, Shamir proposed a new concept, identity-based cryptosystem, to solve the authentication problem of standard public-key cryptosystems [15]. Here the authentication problem is: a public-key file managed by a trusted authority or authority's certificates of public-keys should be employed in order to check the validity of public-keys (i.e., to check the correct linkage between a public-key and a user).

In the identity-based cryptosystem, there exits an authority, who generates users' secret keys by using users's identities and the authority's secret key, and distributes them to users through secure channels. When user $A$ with $A$ 's secret key, $s_{A}$, wants to send a ciphertext to $B$, user $A$ encrypts the message, $m$, into a ciphertext, $c$, using $B$ 's identity, $I D_{B}$, and $s_{A}$, in place of $B$ 's public-key. Since $B$ 's identity can be assumed to be publicly known (i.e., to be known by $A)$, such an identity-based cryptosystem does not require $A$ to interact with any party to get information regarding $B$ (e.g., $B$ 's public-key) when $A$ sends 
a ciphertext to $B$ (i.e., $A$ needs no interaction with any party, after $A$ gets $s_{A}$ from the authority), while $B$ 's public-key should be transmitted to $A$ through a public-key file or directly from $B$, in the standard public-key cryptosystems. Note that the security of identity-based cryptosystems depends much more on the security of the authority than the standard public-key cryptosystems, since the authority knows all users' secret keys.

Although Shamir gave a concrete realization of identity-based digital signature schemes, he left it open as to how to realize identity-based cryptosystems (or identity-based key-distributions). Several schemes have been proposed regarding this problem, but no satisfactory solution to Shamir's open problem has been proposed so far.

\subsection{Trial}

We obtained a solution to this open problem, by using the trapdoor technique based on anomalous elliptic curves. The identity-based cryptosystem (keydistribution) has the following properties:

- This scheme has no interaction and no threshold of collusion (i.e., truly follows Shamir's concept).

- The required computational power for the authority is feasible, i.e., $O\left(|n|^{3}\right)$ per one user key generation.

- The security assumption is clear and feasible: the security of the system is reduced to the intractability of a reasonable primitive problem, the anomalous $E_{n}$-Diffie-Hellman problem, under the random oracle model .

A rough idea of our system is as follows: $n$ and (parameters of) $E_{n}$ are the public system parameters and $(p, q)$ is the secret of the authority, who can compute the discrete logarithm, $s_{A}$, using the SSSA algorithm, where $I D_{A}$ is the identity of user $A, G$ and $F\left(I D_{A}\right)$ are points on $E_{n}$ (i.e., $F$ is a function to embed $I D_{A}$ to a point on $\left.E_{n}\right)$ and $s_{A} G=F\left(I D_{A}\right)$. The authority sends $s_{A}$ to user $A$ secretly, and $A$ can compute a shared key, $K_{A B}$, with user $B$ by using $s_{A}$ and $F\left(I D_{B}\right)$ such that $K_{A B}=s_{A} F\left(I D_{B}\right)=s_{A}\left(s_{B} G\right)=s_{B}\left(s_{A} G\right)=s_{B} F\left(I D_{A}\right)$. Therefore, $B$ can also compute the same key, $K_{A B}$, from $s_{B}$ and $F\left(I D_{A}\right)$. Here note that the authority with secret-key $(p, q)$ can compute $s_{A}$ efficiently (with running time of $O\left(|p|^{3}\right)$ ) using the SSSA algorithm, while it is considered to be hard to compute it without knowing $(p, q)$.

\subsection{Failure}

Unfortunately we have found that the anomalous $E_{n}$-Diffie-Hellman problem is not secure (namely, our scheme is not secure). We have also found an another example of insecure cryptosystems based on anomalous curves. We will observe why the anomalous $E_{n}$-Diffie-Hellman problem is not secure and why it is hard to utilize anomalous curves for realizing a secure cryptographic scheme.

\subsection{Reductions}

Through the observation on our algorithm of breaking the anomalous $E_{n}$-DiffieHellman problem and on the reason why it is hard to utilize anomalous curves 
for constructing secure cryptographic schemes, we have realized a relationship between the breaking algorithm and some reductions.

For this purpose, we will show reductions of factoring $n$ to computing the order of an elliptic curve over $\mathbf{Z} / n \mathbf{Z}$. (See the footnote in Abstract for the meaning of "order".) These reductions roughly imply the equivalence of intractability between factoring and computing elliptic curve's order. Here, the algorithm of breaking our ID-based cryptosystem is considered to be a special case of these reductions, since the order of $E_{n}$ in our identity-based cryptosystem (i.e., in the anomalous $E_{n}$-Diffe-Hellman problem) is trivial, i.e., $n$. The reductions imply that factoring $n$ is trivially easy if and only if the order of $E_{n}$ is trivially obtained.

We will show two types of reductions: one (Reduction 1) is the reduction of the elliptic curve factoring problem (i.e., given $\left(E_{n}, n\right)$, factoring $n$ ) to computing the order of an elliptic curve over $\mathbf{Z} / n \mathbf{Z}$; the other (Reduction 2 ) is the reduction of the factoring problem (given $n$, factoring $n$ ) to computing the order of an elliptic curve over $\mathbf{Z} / n \mathbf{Z}$.

In Reduction 1 , we assume that $E_{n}$ is selected under the condition that $\# E_{p}$ and $\# E_{q}$ have large prime factors. (Note that $n$ can be trivially factored from $E_{n}$ if either \# $E_{p}$ or \# $E_{q}$ has only small prime factors.) The cryptographic implication of this reduction is: When $E_{n}$ is selected by a key generator of a cryptographic scheme (i.e., the key generator checks whether \# $E_{p}$ and \# $E_{q}$ have large prime factors), computing the order of $E_{n}$, given $\left(E_{n}, n\right)$, is as hard as the elliptic curve factoring problem. On the other hand, in Reduction 2 , we assume that $E_{n}$ is randomly selected from a non-negligible fraction of elliptic curves over $\mathbf{Z} / n \mathbf{Z}$. The cryptographic implication of this reduction is: When $E_{n}$ is randomly selected from a non-negligible fraction, computing the order of $E_{n}$, given $\left(E_{n}, n\right)$, is as hard as the factoring problem.

If $E_{n}$ is selected under special cryptographic conditions (or selected from a negligible fraction of elliptic curves over $\mathbf{Z} / n \mathbf{Z}$ ), then only Reduction 1 works, but gives no reduction from the factoring problem. Since $E_{n}$ for our ID-based cryptosystem ( $E_{p}$ and $E_{q}$ are anomalous) is specific (i.e., is selected from a negligible fraction), the breaking algorithm is a special case of Reduction 1, but not of Reduction 2.

\section{Remark (Related Works)}

Miller and Long showed the reduction of factoring $\boldsymbol{n}$ to computing the Euler function $\phi(n)[9,6]$. Kunihiro and Koyama have recently presented the reduction (say the "KK reduction") of the factoring problem to computing the order of an elliptic curve, $E_{n}$, over $\mathbf{Z} / n \mathbf{Z}$ [5]. Hence our Reduction 2 corresponds to the KK reduction. In addition Reduction 1 corresponds to the reduction as a variant of the KK reduction described in Remarks (3), in [5]. Both the KK reduction and our Reduction 2 work for non-negligible fractions of $\# E_{n}$. The difference between these reductions are the failure cases of reduction. The $\mathrm{KK}$ reduction does not work when the order of \# $E_{n}$ has only large prime factors, while our Reduction 2 works for any non-negligible fractions. On the other hand, our Reduction 2 does not consider the case where $n$ has more than two prime factors, while the KK reduction carefully considers such a case. Therefore, for some cases the KK reduction works while our Reduction 2 does not work, and for the other cases our Reduction 2 works while the KK reduction does not work. This difference depends on the underlying techniques in the reductions: $x$-coordinate addition formula and twist curve technique play essential roles in our Reductions 1 and 2 . 


\section{Anomalous Elliptic Curves and the Discrete Logarithm Algorithm}

This section introduces the underlying key techniques and notations employed in our scheme: anomalous elliptic curves, the SSSA algorithm, elliptic curves over $\mathbf{Z} / n \mathbf{Z}$, and the addition formula for $x$-coordinates.

\subsection{Anomalous Elliptic Curves}

Let $K$ be a finite field with characteristics $\neq 2,3$.

An elliptic curve over $K$ (in affine coordinates), denoted by $E(K)$, is the set of all solutions $(x, y) \in K \times K$ to the equation

$$
E: y^{2}=x^{3}+a x+b \quad\left(a, b \in K, 4 a^{3}+27 b^{2} \neq 0\right),
$$

together with a special point $\mathcal{O}_{p}$, called the point at infinity.

The set forms a finite Abelian group, and the group law formula [16](usually we call it the addition, and use the notation + ) is defined over the points on $K$.

Let $p$ be a prime $(p>5)$, and $\mathbf{F}_{p}$ be a finite field with $p$ elements. Now, let $\# E\left(\mathbf{F}_{p}\right)$ be the number of points on $E\left(\mathbf{F}_{p}\right)$, and \#E( $\left.\mathbf{F}_{p}\right)=p+1-t$, where $t$ is called trace and $-2 \sqrt{p} \leq t \leq 2 \sqrt{p})$. An elliptic curve, $E\left(\mathbf{F}_{p}\right)$, is called anomalous if $\# E\left(\mathbf{F}_{p}\right)=p$ (i.e., trace $t$ is equal to 1.)

When $G$ is a point on $E\left(\mathbf{F}_{p}\right), m G$ means $m$ times (elliptic curve) addition of $G$ (i.e., $m G=G+\cdots+G(m$ times $)$ ). Let $G=(x, y) \in E\left(\mathbf{F}_{p}\right)$, then $m G$ is denoted by $(m * x, m * y) \in E\left(\mathbf{F}_{p}\right)$. (i.e., $m * x$ means the $x$-coordinate of $m G$.)

An efficient way to find the parameters of an anomalous elliptic curve over a prime field has been shown by [10].

\subsection{Discrete Logarithm Algorithm of Anomalous Elliptic Curves}

Very recently, Semaev [14], Smart [17] and Satoh and Araki [12] independently discovered an efficient $\left(O\left(|p|^{3}\right)\right.$ algorithm for computing the discrete logarithm for anomalous elliptic curve $E\left(\mathbf{F}_{p}\right)$.

Definition 1. Let $E\left(\mathbf{F}_{p}\right)$ be an anomalous elliptic curve, and $G, P$ be points on $E\left(\mathbf{F}_{p}\right)$. Given, $G, P$, and $E\left(\mathbf{F}_{p}\right)$, the discrete logarithm problem over $E\left(\mathbf{F}_{p}\right)$ is to find integer $m(0 \leq m<p)$ such that $P=m G$.

Although Semaev's algorithm has a different flavor from the algorithm by Smart, Satoh, and Araki, in this paper we introduce Smart-Satoh-Araki's approach and call it the SSSA algorithm as follows (we mainly follow the notations of [12]):

\section{[SSSA Algorithm]}

Input: $G, P$, and $E\left(\mathbf{F}_{p}\right)$

Output: $m$

1. Find a curve, $E_{p^{2}}$, over $\mathbf{Z}$ lifted from $E\left(\mathbf{F}_{p}\right)$, through which non-zero map $\lambda_{E_{p^{2}}}: E\left(\mathbf{F}_{p}\right) \mapsto \mathbf{F}_{p}$ is constructed. (Such a curve can be found efficiently $\left(O\left(|p|^{2}\right)\right)$ by Theorem 3.7 in [12].) 
2. Let $\lambda_{E_{p^{2}}}$ be non-zero. Compute $\lambda_{E_{p^{2}}}(G)$ and $\lambda_{E_{p^{2}}}(P)$. (The computational complexity of computing $\lambda_{E_{p^{2}}}(G)$ is $O\left(|p|^{3}\right)$.) Compute $s=\lambda_{E_{p^{2}}}(P) / \lambda_{E_{p^{2}}}(G)$ $\bmod p$.

\section{[Computation of non-zero map $\lambda_{E_{\mathrm{p}^{2}}}$ ]}

Input: $\alpha=(s, t) \in E\left(\mathbf{F}_{p}\right) \backslash\left\{\mathcal{O}_{p}\right\}, E\left(\mathbf{F}_{p}\right), E_{p^{2}}$

Output: $\lambda_{E_{p^{2}}}(\alpha)$

1. Find $A=(x, y) \in E_{p^{2}}\left(\mathbf{Z} / p^{2} \mathbf{Z}\right)$ satisfying $x \bmod p=s, y \bmod p=t$.

2. Compute $((p-1) * x,(p-1) * y)=(p-1) A_{p} \in E_{p^{2}}\left(\mathbf{Z} / p^{2} \mathbf{Z}\right)$.

3. Compute $\lambda_{E_{p^{2}}}(\alpha)$ as follows:

$$
\lambda_{E_{p^{2}}}(\alpha)=\frac{1}{p} \frac{(p-1) * x-x}{(p-1) * y-y} \bmod p
$$

\subsection{Addition Formula with $x$-coordinates}

Here we show an addition formula only with $x$-coordinates, based on the division polynomials [2].

For $E\left(\mathbf{F}_{p}\right)\left(\left(a_{p}, b_{p}\right)\right.$ be the parameters of $\left.E\left(\mathbf{F}_{p}\right)\right), P=\left(x_{p}, y_{p}\right)$, and $\left(i * x_{p}, i *\right.$ $\left.y_{p}\right)=i P$, if $i * y_{p} \not \equiv 0 \quad(\bmod p)$, then

$$
2 i * x_{p}=\frac{\left(\left(i * x_{p}\right)^{2}-a_{p}\right)^{2}-8 b_{p}\left(i * x_{p}\right)}{4\left(\left(i * x_{p}\right)^{3}+a_{p}\left(i * x_{p}\right)+b_{p}\right)} \bmod p .
$$

In addition, if $i * x_{p} \not \equiv(i+1) * x_{p} \quad(\bmod p)$ and $x_{p} \not \equiv 0 \quad(\bmod p)$, then

$$
(2 i+1) * x_{p}=\frac{\left(a_{p}-\left(i * x_{p}\right)\left((i * 1) * x_{p}\right)\right)^{2}-4 b_{p}\left(i * x_{p}+(i+1) * x_{p}\right)}{x_{p}\left(i * x_{p}-(i+1) * x_{p}\right)^{2}} \quad(\bmod p) .
$$

\subsection{Elliptic Curves over the Ring}

An addition formula with $x$-coordinates on $E(\mathbf{Z} / n \mathbf{Z})$ can be also defined in the same way as the addition formula on $E\left(\mathbf{F}_{p}\right)$. Hence, given $x \in \mathbf{Z} / n \mathbf{Z}$ with $P=(x, y) \in E(\mathbf{Z} / n \mathbf{Z})$, for any integer $m \in \mathbf{Z} / n \mathbf{Z}, m * x$ can be calculated in the running time of $O\left(|n|^{3}\right)$ through the addition chain of the above-mentioned addition formula with $x$-coordinates.

Using the Chinese remainder theorem, every element $c$ of $\mathbf{Z} / n \mathbf{Z}$ can be represented uniquely as a pair $\left[c_{p}, c_{q}\right]$, where $c_{p}=c \bmod p$ and $c_{q}=c \bmod q$. Thus every point $P=(x, y)$ on $E(\mathbf{Z} / n \mathbf{Z})$ can be represented uniquely as a pair $\left[P_{p}, P_{q}\right]=\left[\left(x_{p}, y_{p}\right),\left(x_{q}, y_{q}\right)\right]$, where $P_{p} \in E\left(\mathbf{F}_{p}\right)$ and $P_{q} \in E\left(\mathbf{F}_{q}\right), x=\left[x_{p}, x_{q}\right]$, and $y=\left[y_{p}, y_{q}\right]$. Here we denote $E(\mathbf{Z} / n \mathbf{Z})=\left[E\left(\mathbf{F}_{p}\right), E\left(\mathbf{F}_{q}\right)\right]\left(\right.$ or $\left.E_{n}=\left[E_{p}, E_{q}\right]\right)$, where parameters, $a$ and $b$, of $E(\mathbf{Z} / n \mathbf{Z})$ satisfy the following: $a=\left[a_{p}, a_{q}\right]$, $b=\left[b_{p}, b_{q}\right],\left(a_{p}, b_{p}\right)$ be the parameters of $E\left(\mathbf{F}_{p}\right)$, and $\left(a_{q}, b_{q}\right)$ be the parameters of $E\left(\mathbf{F}_{q}\right)$. 


\section{The Proposed Non-interactive Identity-Based Key-Distribution Scheme}

\subsection{The Proposed Scheme}

The proposed system consists of the trusted authority, $T$, and users, $A, B$ etc.. Authority $T$ generates $T$ 's secret-key, $\boldsymbol{s}_{T}$, and publishes system public parameters. $T$ generates user $A$ 's secret key, $s_{A}$, from $A$ 's identity, $I D_{A}$, by using $s_{T}$, and sends $s_{A}$ to $A$ through a secure channel. User $A$ can non-interactively generate a shared secret key with user $B, K_{A B}$, by using $A$ 's secret key $s_{A}$ and $B$ 's identity, $I D_{B}$. Then, when user $A$ sends a ciphertext to $B$, user $A$ encrypts message $m$ to ciphertext $c=E n c\left(m, K_{A B}\right)$, where $E n c$ is an arbitrary secret-key encryption function with secret-key $K_{A B}$.

Set-up procedure Authority $T$ generates primes $p$ and $q$, and (parameters, $\left(a_{p}, b_{p}\right)$ and $\left(a_{q}, b_{q}\right)$, of $)$ anomalous elliptic curves, $E_{p}$ and $E_{q}$, where $\# E_{p}=p$ and $\# E_{q}=q$. Without loss of generality, we assume $p>q$.

$T$, then, generates and publishes $n=p q$, and (parameters, $(a, b)$, of) $E_{n}=$ $\left[E_{p}, E_{q}\right]$ (i.e., $a \equiv a_{p} \quad(\bmod p), a \equiv a_{q} \quad(\bmod q), b \equiv b_{p} \quad(\bmod p)$, and $b \equiv b_{q}$ $(\bmod q))$.

$T$ also determines points, $G_{p}$ and $G_{q}$, on $E_{p}$ and $E_{q}$, respectively, such that $G_{p} \neq \mathcal{O}_{p}, G_{q} \neq \mathcal{O}_{q} . T$, then, finds non-zero maps, $\lambda_{E_{p^{2}}}: E_{p}\left(\mathbf{F}_{p}\right) \mapsto \mathbf{F}_{p}$ and $\lambda_{E_{q^{2}}}: E_{q}\left(\mathbf{F}_{q}\right) \mapsto \mathbf{F}_{q}$. T publishes $x$-coordinate, $X_{G_{n}}$, of $G_{n}=\left[G_{p}, G_{q}\right]$.

Therefore, the public and secret parameters are as follows:

- [Public system parameters] $n, E_{n}, X_{G_{n}}$

- [Secret key of authority $T] p, q$

Secret key generation for each user Let $h$ be a random function, $h$ : $\{0,1\}^{*} \mapsto\{0,1\}^{k-1}$. (See [1] for the definition of a random function. Practically, we can replace it by a practical one-way function such as SHA and MD5.) Authority $T$ finds the smallest integer $r_{A}\left(r_{A} \geq 0\right)$ such that $h\left(I D_{A} \| r_{A}\right)$ is the $x$-coordinate of a point on $E_{n}$. Let $F$ be a point on $E_{n}$ whose $x$-coordinate is $h\left(I D_{A} \| r_{A}\right)$ (there exist four possible solutions of $F$ and one of them is randomly assigned as $F$ ), and $F_{p}=F \bmod p$ and $F_{q}=F \bmod q$.

$T$ then calculates $s_{A}=\left[s_{A, p}, s_{A, q}\right]$ such that

$$
s_{A, p} G_{p}=F_{p}, \quad s_{A, q} G_{q}=F_{q},
$$

using the SSSA algorithm.

$T$ sends $\left(s_{A}, r_{A}\right)$ to $A$. $A$ checks whether $h\left(I D_{A} \| r_{A}\right)=s_{A} * X_{G_{n}}$ holds (using the addition formula of subsection 2.3) and $r_{A}$ is the smallest non-zero integer such that $n * h\left(I D_{A} \| r_{A}\right)$ is $\infty$, and accepts $\left(s_{A}, r_{A}\right)$ as $A$ 's secret key, if it holds.

(Non-interactive) Key distribution between users Suppose that users $A$ and $B$ want to share a secret key, $K_{A B}$.

User $A$ finds the smallest integer $r_{B}\left(r_{B} \geq 0\right)$ such that $h\left(I D_{B} \| r_{B}\right)$ is the $x$-coordinate of a point on $E_{n}$, by checking whether $\left.\left(\left(h\left(I D_{B} \| r_{B}\right)\right)^{3}+a\left(h\left(I D_{B} \| r_{B}\right)\right)+b\right) / n\right)=1$, and $n * h\left(I D_{B} \| r_{B}\right)=\infty$.

$A$ then calculates $K_{A B}$ as follows:

$$
K_{A B}=s_{A} * h\left(I D_{B} \| r_{B}\right) .
$$




\subsection{Security Condition}

Definition 2. Let $E_{p}\left(\mathbf{F}_{p}\right)$ and $E_{q}\left(\mathbf{F}_{q}\right)$ be anomalous elliptic curves, $E_{n}(\mathbf{Z} / n \mathbf{Z})$ $=\left[E_{p}\left(\mathbf{F}_{p}\right), E_{q}\left(\mathbf{F}_{q}\right)\right]$, and $G$ be a point on $E_{n}(\mathbf{Z} / n \mathbf{Z})$ such that $n=p q(p, q$ : primes $), G=\left[G_{p} \in E_{p}\left(\mathbf{F}_{p}\right), G_{q} \in E_{q}\left(\mathbf{F}_{q}\right)\right]$, and $G_{p} \neq \mathcal{O}_{p}, G_{q} \neq \mathcal{O}_{q}$. Given, $n$, $E(\mathbf{Z} / n \mathbf{Z})$, the $x$-coordinates of $G, s G$, and $t G\left(s, t\right.$ : integers), (say $X_{G}, X_{s G}$, and $\left.X_{t G}\right)$, the anomalous $E_{n}$-Diffie-Hellman problem is to find the $x$-coordinate of point $(s t) G, X_{(s t) G}$.

Definition 3. Let $\mathcal{G}\left(1^{k}\right) \rightarrow\left(n, E(\mathbf{Z} / n \mathbf{Z}), X_{G}, X_{s G}, X_{t G}\right)$, where $k=|n|$, be the parameter generator such that the distribution of $\left(n, E(\mathbf{Z} / n \mathbf{Z}), X_{G}, X_{s G}, X_{t G}\right)$ is the same as that of $\left(n, E_{n}, X_{G_{n}}, h\left(I D_{A} \| r_{A}\right), h\left(I D_{A} \| r_{A}\right)\right)$ of the proposed scheme. (Note that $0 \leq X_{s G}<2^{k-1}, 0 \leq X_{t G}<2^{k-1}$ ) The anomalous $E_{n^{-}}$ Diffie-Hellman problem is intractable if, for any probabilistic polynomial time machine, $M_{0}$, for any constant $c$, for all sufficiently large $k$,

$$
\operatorname{Pr}\left[M_{0}\left(n, E(\mathbf{Z} / n \mathbf{Z}), X_{G}, X_{s G}, X_{t G}\right) \rightarrow X_{(s t) G}\right]<1 / k^{c} .
$$

The probability is taken over the coin flips of $\mathcal{G}$ and $M_{0}$.

Definition 4. Let $\mathcal{G}^{\prime}$ be a generator regarding the proposed scheme such that $\mathcal{G}^{\prime}\left(1^{k}\right) \rightarrow\left(n, E(\mathbf{Z} / n \mathbf{Z}), X_{G_{n}}\right),\left(n, E(\mathbf{Z} / n \mathbf{Z}), X_{G_{n}}\right)$ is public system parameters (with the same distribution of the proposed scheme), and $k=|n|$. Let $I D_{A}$ and $I D_{B}$ be given.

Let $A d v$ be an adversary of the proposed scheme. $A d v$ provides arbitrary $I D_{1}, \ldots, I D_{l}$ (they are not $I D_{A}$ nor $\left.I D_{B}\right)$ to the authority, and obtains $\left(s_{1}, r_{1}\right)$, $\ldots,\left(s_{l}, r_{l}\right)$, such that $h\left(I D_{i} \| r_{i}\right)=s_{i} * X_{G_{n}}$. Adv then tries to find $K_{A B}$ such that $K_{A B}=s_{A} * h\left(I D_{B} \| r_{B}\right)$, and $h\left(I D_{A} \| r_{A}\right)=s_{A} * X_{G_{n}}$.

The proposed scheme is secure if, for any probabilistic polynomial time adversary, $A d v$, for any constant $c$, for all sufficiently large $k$,

$$
\operatorname{Pr}\left[\operatorname{Adv}\left(n, E(\mathbf{Z} / n \mathbf{Z}), X_{G_{n}}, I D_{A}, I D_{B},\left(s_{1}, r_{1}\right), \ldots,\left(s_{l}, r_{l}\right)\right) \rightarrow K_{A B}\right]<1 / k^{c} .
$$

The probability is taken over the coin flips of $\mathcal{G}^{\prime}$, random oracle $h$, and $A d v$.

Theorem 5. Assuming that $h:\{0,1\}^{*} \mapsto\{0,1\}^{k-1}$ is a random function, where $k=|n|$, the proposed scheme is secure if and only if the anomalous $E_{n}$-DiffieHellman problem is intractable.

See Appendix A for the proof.

\section{Anomalous $E_{n}$-Diffie-Hellman Problem is Tractable}

Now we show that the anomalous $E_{n}$-Diffie-Hellman problem is tractable. More precisely, given $\left(n, E_{n}\right)$, there is an efficient algorithm to factor $n$.

Let $E_{p}$ and $E_{q}$ be anomalous elliptic curves such that $E_{n}=\left[E_{p}, E_{q}\right]$, and $\overline{E_{p}}$ and $\overline{E_{q}}$ be the twists of $E_{p}$ and $E_{q}$. That is, \#E $=p, \# E_{q}=q$, \# $\overline{E_{p}}=p+2$, and $\# \overline{E_{q}}=q+2$. (see [2] for the twist of an elliptic curve).

When $X$ is a random integer in $\mathbf{Z} / n \mathbf{Z}$, there are four possibilities as follows:

1. $X$ is the $x$-coordinate of a point on $\left[E_{p}, E_{q}\right]$. Then $n * X$ is $\left[\mathcal{O}_{p}, \mathcal{O}_{q}\right]$. 
2. $X$ is the $x$-coordinate of a point on $\left[\overline{E_{p}}, E_{q}\right]$. Then $n * X$ is [ a finite value, $\left.\mathcal{O}_{q}\right]$.

3. $X$ is the $x$-coordinate of a point on $\left[E_{p}, \overline{E_{q}}\right]$. Then $n * X$ is $\left[\mathcal{O}_{p}\right.$, a finite value ].

4. $X$ is the $x$-coordinate of a point on $\left[\overline{E_{p}}, \overline{E_{q}}\right]$. Then $n * X$ is a finite value (i.e., [a finite value, a finite value ]).

In cases 2 and $3, n * X$ can be used for factor $n$, while $n * X$ cannot be used for factor $n$ in cases 1 and 4 . That is, in cases 2 and $3, \operatorname{gcd}(d, n)$ should be $p$ or $q$, where $d$ be the denominator of $n * X$.

Hence, given $(n, E(\mathbf{Z} / n \mathbf{Z}))$, if $X$ is randomly selected from $\mathbf{Z} / n \mathbf{Z}, n$ can be factored with probability $1 / 2$. By repeating this procedure many $(\operatorname{poly}(|n|))$ times, the success probability of factoring $n$ is overwhelming.

\section{$5 \quad$ Another Trial and Failure}

We can easily construct an elliptic curve version of a public-key cryptosystem [11]. Here, [11] is constructed on the multiplicative group over ring $\mathbf{Z} / n \mathbf{Z}(n=$ $p^{2} q ; p, q$ : primes), while the elliptic curve version is constructed on elliptic curves, $E_{n}$, over ring $\mathbf{Z} / n \mathbf{Z}\left(n=p q ; p, q\right.$ : primes), $E_{n}=\left[E_{p}, E_{q}\right], E_{p}$ is anomalous elliptic curve over $\mathbf{F}_{p}$ and $E_{q}$ is not an anomalous elliptic curve over $\mathbf{F}_{q}$. The $p$-Sylow group in [11] corresponds to the anomalous curve, $E_{p}$.

Obviously $n$ can be easily factored from the parameter of $E_{n}$ in a manner similar to Section 4 (see Appendix B for the related reductions).

\section{Reductions}

Through the observation on our algorithm of breaking the anomalous $E_{n}$-DiffieHellman problem and on the reason why it is hard to utilize anomalous curves for constructing secure cryptographic schemes, we have realized a relationship between the breaking algorithm and some reductions.

For this purpose, this section will show two reductions (Reductions 1 and 2) of factoring $n$ to computing the order of an elliptic curve over $\mathbf{Z} / n \mathbf{Z}$.

We will also show other reductions which are related to the attack introduced in Section 5 in Appendix B.

The algorithms of our reductions are based on the algorithm of breaking our identity cryptosystem (i.e., the anomalous $E_{n}$-Diffie-Hellman problem). Hence this section follows the notations and techniques of Section 4 and the previous sections (e.g., $\overline{E_{p}}$ for the twist curve of elliptic curve $E_{p}$, and $*$ for the elliptic curve addition operation with only $x$-coordinates).

Definition 6. Let $G_{1}$ be an instance generator such that $G_{1}\left(1^{k}\right) \rightarrow n, n=p q$, $|n|=k,(p, q$ : primes, $|p|=|q|)$, where $p$ and $q$ are independently selected. The "factoring problem" is, given $(n, k)$ by $G_{1}$, to find $(p, q)$.

The factoring problem is intractable, if for any probabilistic polynomial time machine $A d v$, for any constant $c$, for sufficiently large $k$,

$$
\operatorname{Pr}\left[\operatorname{Adv}\left(1^{k}, n\right)=(p, q)\right]<1 / k^{c} .
$$

The probability is taken over the coin flips of $G_{1}$ and $A d v$. 
Definition 7. Let $G_{2}$ be an instance generator such that $G_{2}\left(1^{k}\right) \rightarrow\left(E_{n}, n\right)$, $n=p q,|n|=k(p, q$ : primes, $|p|=|q|), E_{n}=\left[E_{p}, E_{q}\right]\left(E_{p}\right.$ and $E_{q}$ are elliptic curves over $\mathbf{F}_{p}$ and $\mathbf{F}_{q}$, respectively). Here $p$ and $q$ are independently selected.

The "elliptic curve factoring problem" is, given $\left(E_{n}, n, k\right)$ by $G_{2}$, to find $(p, q)$.

The elliptic curve factoring problem is intractable, if for any probabilistic polynomial time machine $A d v$, for any constant $c$, for sufficiently large $k$,

$$
\operatorname{Pr}\left[\operatorname{Adv}\left(1^{k}, E_{n}, n\right)=(p, q)\right]<1 / k^{c} .
$$

The probability is taken over the coin flips of $G_{2}$ and $A d v$.

Definition 8. Let $G_{3}$ be an instance generator such that $G_{3}\left(1^{k}\right) \rightarrow\left(E_{n}, n\right)$, $n=p q,|n|=k(p, q$ : primes, $|p|=|q|), E_{n}=\left[E_{p}, E_{q}\right]$. Here, $p$ and $q$ are independently selected.

The "elliptic curve order problem" is, given $\left(E_{n}, n, k\right)$, to find $\Phi\left(E_{n}\right)$, where $\Phi\left(E_{n}\right)=\operatorname{lcm}\left(\# E_{p}, \# E_{q}\right)$.

The elliptic curve order problem is intractable, if for any probabilistic polynomial time machine $A d v$, for any constant $c$, for sufficiently large $k$,

$$
\operatorname{Pr}\left[\operatorname{Adv}\left(1^{k}, E_{n}, n\right)=\Phi\left(E_{n}\right)\right]<1 / k^{c} .
$$

The probability is taken over the coin flips of $G_{3}$ and $A d v$.

Theorem 9. [Reduction 1] Let $G_{3}$ for the elliptic curve order problem be equivalent to $G_{2}$ for the elliptic curve factoring problem. Assume $G_{2}$ and $G_{3}$ output $E_{n}$ such that \# $E_{p}$ and \# $E_{q}$ have large prime factors, $p^{\prime}$ and $q^{\prime}$, respectively, i.e., \# $E_{p}=p^{\prime} u, \# E_{q}=q^{\prime} v, 1 \leq u<q^{\frac{1}{4}}, 0 \leq v<q^{\frac{1}{4}}$.

Then, the elliptic curve order problem is intractable if and only if the elliptic curve factoring problem is intractable.

\section{Proof. (Only if:)}

Assume that the elliptic curve factoring problem is not intractable. That is, an adversary, $A d v$, given $\left(E_{n}, n, k\right)$, can factor $n$ with non-negligible probability. We will then construct a polynomial time machine $M$ with help of $A d v$ (with knowing $(p, q))$, which, given $\left(E_{n}, n, k\right)$, computes $\Phi\left(E_{n}\right)$ with non-negligible probability. $M$ obtains elliptic curves $E_{p}$ and $E_{q}$ such that $E_{n}=\left[E_{p}, E_{q}\right] . M$ computes \# $E_{p}$, \# $E_{q}$ with using Schoof's algorithm [13], whose running time is of the order of polynomial. So, $M^{A d v}$ can compute $\Phi\left(E_{n}\right)=\operatorname{lcm}\left(\# E_{p}, \# E_{q}\right)$ with non-negligible probability. (If:)

Let assume that the elliptic curve order problem is not intractable. That is, an adversary, $A d v$, can compute $\Phi\left(E_{n}\right)$ from $\left(E_{n}, n, k\right)$ with non-negligible probability. We will then construct a probabilistic polynomial time algorithm $M$, with help of $A d v$ as an oracle, which, given $\left(E_{n}, n, k\right)$, can factor $n$ with non-negligible probability.

When $M$ randomly selects $z \in \mathbf{Z} / n \mathbf{Z},\left(\frac{f(z)}{n}\right)=-1$ with probability $1 / 2$, where $f$ denotes a cubic polynomial which defines the elliptic curve $E_{n}=\left[E_{p}, E_{q}\right]$ and $\left(\frac{f(z)}{n}\right)$ the Jacobi symbol.

Then we have two possibilities: $z$ is the $x$-coordinate of a point on $\left[E_{p}, \bar{E}_{q}\right]$ and is the $x$-coordinate of a point on $\left[\bar{E}_{p}, E_{q}\right]$, where $\bar{E}_{q}$ (and $\bar{E}_{p}$ ) is the twist of 
$E_{q}\left(\right.$ and $\left.E_{p}\right)$ [2], and $\# \bar{E}_{q}=q+1+s$ (and \# $\bar{E}_{p}=p+1+t$ ) when \# $E_{q}=q+1-s$ (and $\# E_{p}=p+1-t$ ). Without loss of generality, we assume that the first case occurs.

Since the distribution of $\left(E_{n}, n, k\right)$ is given by $G_{3}=G_{2}, A d v$ can output a correct $\Phi\left(E_{n}\right)$ with non-negligible probability.

Let $a=\operatorname{gcd}(q+1-s, q+1+s), a b=q+1+s$, then, it is easy to see that $q^{\prime} \not q+1+s, a \leq 4 \sqrt{q}$. On the other hand, since $p^{\prime} \not q+1+s$ with overwhelming probability over the distribution of $p^{\prime}, a$ is a divisor of $v$.

Hence, we have $a<q^{\frac{1}{4}}$ and $\operatorname{gcd}(p+1-t, b)=\operatorname{gcd}(u, b)<q^{\frac{1}{4}}$.

In order to evaluate the probability of the case when $\Phi\left(E_{n}\right) * z$ is the $x$ coordinate of $\left[\mathcal{O}_{p}, \mathcal{O}_{q}\right]$, it is sufficient to consider the point of $a \operatorname{gcd}(u, b) * z$.

Let the maximum order of a point on $\bar{E}_{q}\left(\mathbf{F}_{q}\right) \backslash H_{a}$ be $o_{\max }$. Then, $o_{\max }$ is at least $\sqrt{(q+1+s)-\# H_{a}}$ due to the group structure of elliptic curve, where $H_{a}$ denotes the set of $a$-torsion points of $\bar{E}_{q}\left(\mathbf{F}_{q}\right)$. Since \# $H_{a} \leq a^{2}$, we obtain $o_{\max } \geq \sqrt{(q+1+s)-\sqrt{q}}$.

Obviously, $\sqrt{(q+1+s)-\sqrt{q}} \geq \sqrt{q+1-3 \sqrt{q}}>q^{\frac{1}{4}}$, so we have $o_{\max }>q^{\frac{1}{4}}$.

Now, if we choose $z$ randomly such that $z_{q}=z \bmod q$ is not the $x$-coordinate of point $H_{a}$ and has order $o_{m a x}$, then $a \operatorname{gcd}(b, u) * z$ is the $x$-coordinate of $\left[\mathcal{O}_{p}\right.$, a finite value]. We will then evaluate the probability that a randomly selected point, $z_{q}$, is not the $x$-coordinate of a point $H_{a}$ and has order $o_{\max }$.

It is easy to see that there are $\varphi\left(o_{\max }\right)$ many points of order $o_{\max }$. Hence the probability that a randomly selected point, $z_{q}$, is not the $x$-coordinate of a point $H_{a}$ and has order $o_{\text {max }}$ is at least $\frac{1}{6 \log \log \left(q^{1 / 4}\right)}=O\left(\frac{1}{\log k}\right)$.

Hence, $M$ selects polynomially many $z$ randomly, for at least one of them, , $\Phi\left(E_{n}\right) * z$ is the $x$-coordinate of a point, $\left[\mathcal{O}_{p}\right.$, a finite value $]$, with overwhelming probability.

Thus, $M$ can factor $n$ with help of $A d v$ with non-negligible probability.

Theorem 10. [Reduction 2] Let the distribution of $n$ given by $G_{1}$ for the factoring problem be equivalent to that given by $G_{3}$ for the elliptic curve order problem. Let $E_{n}$ be uniformly selected by $G_{3}$ (i.e., the parameters of $E_{n}$ are uniformly and independently selected over $\mathbf{Z} / n \mathbf{Z}$ ). Assume that the distribution of $\# E_{p}$ (and \# $E_{q}$ ) is statistically close to the uniform distribution over $\{0,1\}^{k / 2}$, where the distribution is taken over the coin flips of $G_{3}$ (i.e., random selection of $p, q, E_{p}$ and $\left.E_{q}\right)$.

Then the elliptic curve order problem is intractable if and only of the factoring problem is intractable.

Proof. Since the proof of the only if part is almost trivial, we only give the proof of the if part.

Let assume that the elliptic curve order problem is not intractable, so, there exists an adversary, $A d v$, which can compute $\Phi\left(E_{n}\right)$ from $\left(E_{n}, n, k\right)$ with nonnegligible probability.

Then we will construct a probabilistic polynomial time algorithm $M$, with help of $A d v$ as an oracle, which, given $n$ by $G_{1}$, can factor $n$ with non-negligible probability.

$M$ uniformly selects an elliptic curve $\bmod n, E_{n}$, and randomly chooses $z \in$ $\mathbf{Z} / n \mathbf{Z}$, then $\left(\frac{f(z)}{n}\right)=-1$ with probability $1 / 2$ (see the proof of Theorem 9 for the notations of $f$ and $\left(\frac{f(z)}{n}\right)$ ). 
Then we have two possibilities: $z$ is the $x$-coordinate of a point on $\left[E_{p}, \bar{E}_{q}\right]$ and is the $x$-coordinate of a point on $\left[\bar{E}_{p}, E_{q}\right]$. Without loss of generality, we can assume that the first case occurs.

Since $M$ chooses $E_{n}$ uniformly as $G_{3}$ does, $A d v$ outputs correct $\Phi\left(E_{n}\right)$ with non-negligible probability.

Let $\# E_{p}=p+1-t$ and $\# E_{q}=q+1-s$, therefore $\# \bar{E}_{p}=p+1+t$ and $\# \bar{E}_{q}=q+1+s$. We now evaluate the probability of the case when $\Phi\left(E_{n}\right) * z$ is the $x$-coordinate of $\left[\mathcal{O}_{p}, \mathcal{O}_{q}\right]$ (only in this case $M$ fails in factoring $n$ ).

Let $a=\operatorname{gcd}(q+1-s, q+1+s)$ and $q+1+s=a b$, then it is obvious that $1 \leq a \leq 4 \sqrt{q}$. Hence $b \geq \frac{q+1+s}{4 \sqrt{q}}=O\left(2^{k / 2}\right)$.

First we investigate the maximum size of a square factor of $\# \bar{E}_{q}$. Let $b=$ $c d_{1} d_{2}^{2}$, where $c$ is square free and $d_{1}=\operatorname{gcd}(a, b)$. Let $d=d_{1} d_{2}, l_{1}=\left|\# \bar{E}_{q}\right|, l_{2}=|d|$ and $l_{1}-2 l_{2}=\left|a c / d_{1}\right|$. Since $\# \bar{E}_{q}$ is assumed to be uniformly distributed over $\{0,1\}^{l_{1}}$, the probability, $T$, that $\# \bar{E}_{q}$ has a square factor with size of at least $l_{2}$ bits is

$$
T=\sum_{l_{2} \leq l \leq l_{1} / 2} \frac{2^{l+\left(l_{1}-2 l\right)}}{2^{l_{1}}}=\sum_{l_{2} \leq l \leq l_{1} / 2} \frac{1}{2^{l}} .
$$

Therefore, if $l_{2}>e \log k$ for any constant $e$ and for sufficiently large $k$ (i.e., $2^{l_{2}}$ is super-polynomial), then $T$ is negligible in $k$. Therefore, $|d|=e \log k$ for constant $e$ with overwhelming probability.

Let the maximum order of a point on $\bar{E}_{q}$ be $o_{\bar{E}_{q}}$. Then, $o_{\bar{E}_{q}} / a$ is at least $b / d$ due to the group structure of elliptic curve.

We will then evaluate the probability that $b / d$ divides $\# E_{p}=(p+1-t)$. In this case, $\Phi\left(E_{n}\right) * z$ is the $x$-coordinate of $\left[\mathcal{O}_{p}, \mathcal{O}_{q}\right]$, when $o_{\bar{E}_{q}} / a=b / d$. Since we assume \# $E_{p}$ is distributed statistically uniformly and independently from \# $E_{q}$ (i.e., $b / d)$, the probability that $b / d$ divides $\# E_{p}=(p+1-t)$ is $\frac{d}{b}=O\left(\frac{k^{e}}{2^{k / 2}}\right)$, which is negligible in $k$.

If $b / d$ does not divide $\# E_{p}=(p+1-t)$, then $\Phi\left(E_{n}\right) * z$ is the $x$-coordinate of a point, $\left[\mathcal{O}_{p}\right.$, a finite value], for a point, $z$, such that $z_{q}=z \bmod q$ has the maximum order on $\bar{E}_{q}$. The probability that a randomly selected point, $z_{q}$, has the the maximum order is $\frac{1}{6 \ln \ln q+1+s}=O\left(\frac{1}{\log k}\right)$.

Hence, when $M$ selects polynomially many $z$ randomly, for at least one of them, $\Phi\left(E_{n}\right) * z$ is the $x$-coordinate of a point, [ $\mathcal{O}_{p}$, a finite value], with overwhelming probability. ability.

Thus, $M$ can factor $n$ with help of the oracle, Adv, with non-negligible prob-

\section{Conclusion}

This paper has introduced our trial of realizing an identity-based cryptosystem based on the SSSA algorithm, and then showed that our scheme is not secure. We have generalized the observation of our breaking algorithm and presented reductions of factoring $n$ to computing the order of an elliptic curve over $\mathbf{Z} / n \mathbf{Z}$. Through these reductions, we can clarify the essential reason why our system was broken: $E_{n}$ in our system is a very specific curve such that $\Phi\left(E_{n}\right)$ is known $\left(\Phi\left(E_{n}\right)=n\right)$. 


\section{References}

1. Bellare, M. and Rogaway, P. : Optimal Asymmetric Encryption, Proc. of Eurocrypt'94, LNCS 950, Springer-Verlag pp.92-111 (1995).

2. Demytko, N.: A New Elliptic Curve Based Analogue of RSA, Proc. of Eurocrypt'93, LNCS 765, Springer-Verlag, pp.40-49 (1994).

3. Diffie, W. and Hellman, M.: New Directions in Cryptography, IEEE Trans. on Information Theory, IT-22, 6, pp.644-654 (1976).

4. Fiat, A. and Shamir, A.: How to Prove Yourself: Practical Solutions to Identification and Signature Problems, Proc. of Crypto'86, LNCS 263, Springer-Verlag, pp.186-194 (1986).

5. Kunihiro, N. and Koyama, K.: Equivalence of Counting the Number of Points on Elliptic Curve over the Ring $Z_{n}$ and Factoring $n$, in these proceedings.

6. Long, D.L.: Random Equivalence of Factorization and Computation of Orders, Technical Report 284, Princeton Univ. Dept. of EECS (1981).

7. Maurer, U. and Yacobi, Y.: Non-interactive Public-Key Cryptography, Proc. of Eurocrypt'91, LNCS, Springer-Verlag, pp.498-507 (1992).

8. Maurer, U. and Yacobi, Y.: A Remark on a Non-interactive Public-Key Distribution System, Proc. of Eurocrypt'92, LNCS, Springer-Verlag, pp.458-460 (1993).

9. Miller, G.L.: Riemann's Hypothesis and Tests for Primality, JCSS, 13, pp.300-317 (1976).

10. Miyaji, A.: Elliptic Curves over $F_{p}$ Suitable for Cryptosystems, Proc. of Auscrypt'92, LNCS, Springer-Verlag, pp.479-504 (1993).

11. Okamoto, T. and Uchiyama, S.: A New Public-Key Cryptosystem as Secure as Factoring, in these proceedings.

12. Satoh, T. and Araki, K.: Fermat Quotient and the Polynomial Time Discrete Log Algorithm for Anomalous Elliptic Curves, Preprint (September, 1997).

13. Schoof, R.: Elliptic Curve over Finite Fields and the Computation of Square Roots Mod $p$, Mathematics of Computation, 44, pp.483-494 (1985).

14. Semaev, I.A.: Evaluation of Discrete Logarithms on Some Elliptic Curves, to appear in Mathematics of Computation.

15. Shamir, A.: Identity-Based Cryptosystems and Signature Schemes, Proc. of Crypto'84, LNCS 196, Springer-Verlag, pp.47-53 (1985).

16. Silverman, J.: The Arithmetic of Elliptic Curves, GTM106, Springer-Verlag, 1986.

17. Smart, N.P.: The Discrete Logarithm Problem on Elliptic Curves of Trace One, Preprint (September, 1997).

\section{Appendix A: Proof of Theorem 5}

Since the only-if part is trivial, we will prove the if part. To prove it, assume that the proposed scheme is not secure, i.e., there exists Adv who can break the proposed scheme with non-negligible probability. We will then construct a probabilistic polynomial time algorithm $M$, with the help of $A d v$ as an oracle, which can break the anomalous $E_{n}$-Diffie-Hellman problem with non-negligible probability.

First, $M$ generates $s_{i} \in \mathbf{Z} / n \mathbf{Z}$ uniformly $(i=1, \ldots, l)$, and calculates $X_{i}=$ $s_{i} * X_{G_{n}}$. If $X_{i} \geq 2^{k}$, then discard it and select $s_{i}$ again in the same way. Repeat the procedure until all of $X_{i}(i=1, \ldots, l)$ are within the range of $\{0,1\}^{k}$. Since $2^{k} / n>1 / 2$, the expected number of the repetitions for each $X_{i}$ is less than 2 , and in total, the expected number of the repetitions is less than $2 l$.

$M$ then runs $A d v$ and gives $X_{1}, \ldots, X_{1}$ to $A d v$ along with $\left(r_{1}, \ldots, r_{I}\right)$ as the reply to $A d v$ 's queries to random oracle $h$. Namely, after $A d v$ gives $\left(I D_{1}, \ldots, I D_{l}\right)$ to $M$ as $h, M$ randomly selects $r_{i}$ such that its distribution is the same as that of 
the real system, and $M$ returns $X_{1}, \ldots, X_{l}$ as $h\left(I D_{1} \| r_{1}\right), \ldots, h\left(I D_{l} \| r_{l}\right)$ to $A d v$ along with $\left(r_{1}, \ldots, r_{l}\right)$. Clearly, the distribution of $M$ 's reply is the same as that of the reply by random oracle.

$M$ then gives $\left(s_{1}, \ldots, s_{l}\right)$ to $A d v$ as the authority's reply to $A d v$.

Finally $M$ gives $X_{s G}$ and $X_{t G}$ as $\left(h\left(I D_{A} \| r_{A}\right)\right.$ and $\left(h\left(I D_{B} \| r_{B}\right)\right.$ to $A d v$, and $A d v$ replies $K_{A B}=s_{A} *\left(s_{B} * X_{G_{n}}\right)=X_{(s t) G}$ to $M$ with non-negligible probability, since the distribution of the messages given to $A d v$ by $M$ is exactly the same as that given by $h$ and the authority.

Thus, $M$ with the help of $A d v$ can break the the anomalous $E_{n}$-Diffie-Hellman problem with non-negligible probability.

\section{Appendix B: Another Type of Reductions}

Here we will show another type of reductions, which are related to the attack algorithm shown in Section 5. That is, the attack algorithm is a special case of the reductions in this section.

Definition 11. Let $G_{4}$ be an instance generator such that $G_{4}\left(1^{k}\right) \rightarrow\left(E_{n}, n\right)$, $n=p q,|n|=k(p, q$ : primes, $|p|=|q|), E_{n}=\left[E_{p}, E_{q}\right]$. Here $p$ and $q$ are independently selected.

Let $\Phi\left(E_{p}\right)=m \cdot \# E_{p}$, where $m$ is an integer such that $\frac{\# E_{q}}{\operatorname{gcd}\left(\# E_{p}, \# E_{q}\right)} \not m$ (i.e., $\left.\Phi\left(E_{n}\right) \not \Phi\left(E_{p}\right)\right)$.

The modified elliptic curve order problem is, given $\left(E_{n}, n, k\right)$, to find $\Phi\left(E_{p}\right)$.

The modified elliptic curve order problem is intractable, if for any probabilistic polynomial time machine $A d v$, for any constant $c$, for sufficiently large $k$,

$$
\operatorname{Pr}\left[\operatorname{Adv}\left(1^{k}, E_{n}, n\right)=\Phi\left(E_{p}\right)\right]<1 / k^{c} .
$$

The probability is taken over the coin flips of $G_{4}$ and $A d v$.

Theorem 12. [Reduction 3] Let $G_{4}$ for the modified elliptic curve order problem be equivalent to $G_{2}$ for the elliptic curve factoring problem.

The modified elliptic curve order problem is intractable if and only if the elliptic curve factoring problem is intractable.

Proof. Since the proof of the only if part is almost trivial, we only give the proof of the if part.

Assume that the modified elliptic curve order problem is intractable. That is, there exists an adversary, $A d v$, which can compute $\Phi\left(E_{p}\right)$ from $\left(E_{n}, n, k\right)$ with non-negligible probability. We will then construct a probabilistic polynomial time machine $M$, with help of $A d v$ as an oracle, which given $\left(E_{n}, n, k\right)$, can factor $n$ with non-negligible probability.

Since the distribution of $\left(E_{n}, n, k\right)$ given by $G_{2}$ is equivalent to that by $G_{4}$, $A d v$ outputs a correct $\Phi\left(E_{p}\right)$ with non-negligible probability.

$M$ randomly selects $z \in \mathbf{Z} / n \mathbf{Z}$ such that $\left(\frac{f(z)}{n}\right)=1$ with probability $1 / 2$ (see the proof of Theorem 9 for the notations of $f$ and $\left(\frac{f(z)}{n}\right)$ ).

Then we have two possibilities: $z$ is the $x$-coordinate of a point on $\left[E_{p}, E_{q}\right]$ and is the $x$-coordinate of a point on $\left[\bar{E}_{p}, \bar{E}_{q}\right]$. In the former case, if $z$ is a point such that $z_{q}=z \bmod q$ has the maximum order on $\bar{E}_{q}$, then $\Phi\left(E_{p}\right) * z$ is the $x$-coordinate of $\left[\mathcal{O}_{p}\right.$, a finite value $]$. 
Hence, when $M$ selects polynomially many $z$ randomly, for at least one of them, $\Phi\left(E_{n}\right) * z$ is the $x$-coordinate of a point, $\left[\mathcal{O}_{p}\right.$, a finite value], with overwhelming probability. ability.

Thus, $M$ can factor $n$ with help of the oracle, $A d v$, with non-negligible prob-

Theorem 13. [Reduction 4] Let the distribution of $n$ given by $G_{1}$ for the factoring problem be equivalent to that given by $G_{4}$ for the modified elliptic curve order problem. Let $E_{n}$ be uniformly selected by $G_{4}$ (i.e., the parameters of $E_{n}$ are uniformly and independently selected over $\mathrm{Z} / n \mathrm{Z}$ ).

Then the modified elliptic curve order problem is intractable if and only if the factoring problem is intractable.

Proof. Since the proof of the only if part is almost trivial, we only give the proof of the if part.

Let assume that the modified elliptic curve order problem is not intractable, i.e., there exists an adversary, $A d v$, which computes $\Phi\left(E_{p}\right)$ from $\left(E_{n}, n, k\right)$ with non-negligible probability.

Then we will construct a probabilistic polynomial time algorithm $M$, with help of $A d v$ as an oracle, which, given $n$ by $G_{1}$, can factor $n$ with non-negligible probability.

$M$ uniformly selects an elliptic curve $\bmod n, E_{n}$. Here note that the distribution of $E_{n}$ given by $M$ is the same as that given by $G_{4}$.

The remaining algorithm for this reduction is the same as that of Reduction 3. Thus, $M$ can factor $n$ with help of the oracle, $A d v$, with non-negligible probability. 